A Weight-Related Growth Mindset Increases Negative Attitudes Towards Obese People

Nic Hooper ${ }^{1}$, Alison Crumpton ${ }^{2}$, Michael D. Robinson ${ }^{3}$ and Brian P. Meier ${ }^{4}$

\author{
${ }^{1}$ University of the West of England \\ ${ }^{2}$ University of Warwick \\ ${ }^{3}$ North Dakota State University \\ ${ }^{4}$ Gettysburg College
}

Corresponding author: Nic Hooper, University of the West of England,

nic.hooper@uwe.ac.uk

Running title: Growth mindset and obesity

Acknowledgements: None.

Funding: None. 


\begin{abstract}
In implicit personality theory, people with entity views or a fixed mindset perceive characteristics (e.g., intelligence) as uncontrollable, whereas people with incremental views or a growth mindset perceive characteristics as controllable. In addition to other benefits, the literature sometimes suggests that having a growth mindset will protect against prejudice, which the current two studies examine in terms of negative attitudes towards obese people. Participants (total $N=501$ ) were randomly assigned to complete a questionnaire assessing attitudes towards an obese or non-obese person and a self-theory questionnaire also assessed ideas about body weight. People with a growth mindset, and not fixed mindset, were more likely to have negative attitudes towards obese individuals, pointing to a potential downside of growth mindset in the obesity domain.
\end{abstract}

Key words: mindset, attitudes and obesity. 


\section{A Weight-Related Growth Mindset Increases Negative Attitudes Towards Obese People}

Despite the high prevalence of obesity in the western world (World Health

Organisation, 2013), obese individuals often experience discrimination in the workplace and in their personal lives (Puhl \& Brownell, 2001, 2003a, 2003b). Consistent with such perceptions, the media either under-represents obese people or more frequently makes them objects of ridicule (Puhl \& Brownell, 2003a). Perhaps as a result of such influences, obese people tend to be viewed less favourably by their peers (Goodman, Richardson, Dornbusch, \& Hastorf, 1963) and they encounter dating and marital issues as well (Burnette, 2010).

Crandall (1994) argues that the pervasiveness of negative attitudes towards the obese follows from tendencies to hold people accountable for their behaviours. In a manner in line with the just world hypothesis (Rubin \& Peplau, 1975) or system justification theory (Jost, Banaji, \& Nosek, 2004), perceivers may think that obese people deserve their problems because they do not take proper care of their bodies though they could do so. Puhl and Brownell's (2001) review of obesity biases confirms that such factors are evident in the obesity domain. For example, children who attributed the weight of an obese person to factors within the obese person's control were less favourable toward them and this trend was evident across age groups, independent of the weight of the perceiver (Puhl \& Brownell, 2001; see also Hegarty \& Golden, 2008). As a general principle, people tend to be more sympathetic towards others when their suffering is perceived to be uncontrollable; by contrast, hostility can occur when the same causes are perceived as controllable (Weiner, 1986).

These ideas about controllability are interesting in part because perceptions of control are generally seen to be functional (Bandura, 1989). That is, when people think that they have control over an outcome (versus no control or lesser control), they are more likely to take advantage of the opportunities that they have for making effective changes (Schwarzer, 
2001). Ideas such as these figure prominently in an impressive body of work on implicit personality theory or self-theory (Dweck, 2015), which contrasts entity theorists, who believe that one's standing along a given dimension (e.g., intelligence) is more or less fixed, with incremental theorists, who believe in controllability and the potential for growth (e.g., Biddle, Wang, Chatzisarantis, \& Spray, 2003). A growth mindset, characteristic of incremental theorists, will typically be more conducive to making positive changes over time (Dweck \& Leggett, 1988), including with respect to weight and fitness (Biddle et al., 2003). However, this same mindset could reasonably be linked to blaming other people for their problems, at least under some circumstances. Specifically, if one thinks that characteristics like obesity are controllable, which is more consistent with a growth than fixed mindset, then one may be more inclined to blame others for such problems. Through routes of this type (Weiner, Perry, \& Magnusson, 1988), a generally beneficial sense of growth could plausibly be linked to having negative attitudes towards others who are seen to be responsible for their predicaments.

On the other hand, arguments could also be made that having a growth mindset would promote lesser, rather than greater, negative attitudes. The rationale here begins with the idea that people with a fixed mindset believe that the characteristics a person has will remain with them in the future. If those characteristics are viewed negatively, then the target will be viewed negatively in the future too, evoking dislike and contempt (Dweck \& Leggett, 1988). Conversely, people with a growth mindset should see a greater potential for change among marginalised groups, which might promote more benevolent attitudes. Some research has supported these ideas, suggesting that people with a growth mindset may, at least in certain contexts, be less prejudiced and more compassionate towards stigmatised individuals (Levy, Stroessner, \& Dweck, 1998). 
As of yet, no research has investigated possible links between implicit theories (Dweck, 2015) and negative attitudes towards the obese. Given that perceptions of controllability and blame figure prominently in negative attitudes towards the obese (Crandall, 1994), we thought it likely that people endorsing growth-related ideas about body weight would hold more negative attitudes towards an obese individual than those with a fixed mindset. To investigate this possibility, we assessed lay beliefs in the obesity domain in combination with two person-perception experiments designed to examine attitudes towards obese versus non-obese individuals.

\section{Study 1}

\section{Method}

\section{Participants and Design}

A sample of 171 U.K. participants were recruited via social media (Facebook, Twitter, \& e-mail) and received no compensation for taking part in the study. This data collection route was used both for its convenience and because it tends to result in more diverse samples than the typical university participant pool. Further, data collected from online studies is typically comparable in quality to that collected in the lab (Kraut et al., 2004) and a case could be made that online procedures are useful when sensitive topics are involved. Participants, in a between-subjects design, were directed to receive either the obese or non-obese vignette described below, which was accomplished by randomizing link-picker software. Due to an oversight, information about gender, age, and race were not collected in the first experiment, though we did collect information about height and weight.

\section{Materials and Procedure}

Participants were randomly assigned to read a vignette about either an obese $(N=83)$ or non-obese $(N=88)$ person and then completed a questionnaire designed to gauge their negative attitudes towards a fictional character in a vignette. Participants then completed a 
questionnaire to measure their fixed versus growth mindset in relation to body weight before reporting their own height and weight, the latter to ensure that BMI was similar across vignette conditions.

Vignette. The vignette concerned a fictional character named Betty. In the obese version of this vignette, Betty was described as obese, based on a medical check-up; in the non-obese version of this vignette, the same check-up did not result in this feedback.

Otherwise, the information in the two versions of the vignette was identical and it included some brief mention of family, occupation, and leisure interests (see Appendix).

Assessment of Negative Attitudes. Negative attitudes towards Betty were assessed using a modified version of the Anti-Fat Questionnaire (AFQ: Crandall, 1994). The AFQ is a 12-item questionnaire that asks participants to rate the extent to which they agree/disagree with statements on a 9-point scale $(1=$ strongly agree; $9=$ strongly disagree $)$. In the original questionnaire, the statements focused on overweight people in general (e.g. 'I don't really like fat people much"). In the current context, these items were modified such that they focused on Betty (e.g., "I don't really like Betty much”), and all items were scored such that higher numbers reflected negative attitudes $(M=3.85 ; S D=1.40 ; \alpha=.89)$.

Self-Theories about Weight. While the original Self-Theories Questionnaire (STQ) focused on intelligence (Dweck, 2000), Burnette (2010) created a counterpart centred on beliefs about weight. In both cases, the measure contrasts people with fixed versus malleable beliefs about the attribute (e.g., weight) in question. In specific terms, the weight-based STQ pairs 6 statements (e.g., “you have a certain body weight and you can’t really do much to change it") with an agree/disagree format ( $1=$ strongly agree; $6=$ strongly disagree). We scored this measure such that higher numbers indicate a growth mindset and lower numbers indicate a fixed mindset $(M=4.50 ; S D=0.90 ; \alpha=.87)$.

\section{Results}


We first calculated BMI $(M=23.08 ; S D=3.44 ; 12$ participants did not report height or weight information) to ensure that this pertinent subject characteristic was equivalent across conditions. Weight Condition (vignette character was obese versus not) was not associated with BMI scores in a one-way ANOVA, $F(1,157)=.74, p=.39, \eta^{2}=.01$.

In order to test the main hypothesis, a multiple-regression analysis was performed. The dependent measure was AFQ scores (negative attitudes) and the predictors were Weight Condition, a dichotomous variable, and STQ scores (mindset), a continuous variable. These predictors were Z-scored and an interaction term was also computed (Aiken \& West, 1991). The effect of Weight Condition was significant, $\beta=.19, t(170)=2.71, p<.01$, such that participants had more negative attitudes concerning the obese character $(M=4.11 ; S D=$ 1.56) relative to the non-obese character $(M=3.59 ; S D=1.20)$. There was also an effect for STQ scores, such that people with a growth mindset evaluated the target character more negatively, $\beta=.24, t(170)=3.42, p<.01$.

Of most importance, the interaction between Weight Condition and STQ scores was significant, $\beta=.25, t(170)=3.49, p<.01$. To determine the pattern of this interaction, we computed estimated means by condition at low $(-1 S D)$ versus high $(+1 S D)$ levels of the growth mindset predictor. As shown in Figure 1, the interaction is straightforward: In the obese condition, people with a growth mindset about weight had more negative attitudes towards the obese target character, $\beta=.49, t(170)=4.91, p<.01$. In the non-obese condition, STQ scores did not relate to AFQ scores, $\beta=-.01, t(170)=-.05, p=.96$, as determined by simple slopes analyses (Aiken \& West, 1991). That is, people who viewed weight as controllable (who had a growth mindset concerning weight) expressed greater hostility toward a target person who happened to be obese. 
Additional analyses provided a complementary perspective on the interaction. Weight condition (obese versus non-obese) mattered for people endorsing more of a growth mindset $(+1 S D), \beta=.44, t(170)=4.38, p<.01$, but not for people endorsing more of a fixed mindset $(-1 S D), \beta=-.06, t(170)=-.56, p=.58$.

\section{Discussion and Study 2}

Study 1 found that people with a growth mindset concerning weight, relative to a fixed mindset, expressed more negative attitudes towards an obese person. The finding suggests that people with a growth mindset can be more prejudiced under some circumstances. Nonetheless, a second study was warranted for at least two reasons. The results of Study 1 were novel to the literature and it therefore seemed important to replicate them in a straightforward manner (Simons, 2014). In addition, the participant recruitment method of Study 1 could have led to the inclusion of friends or acquaintances of the authors, which might not be ideal. In Study 2, we fixed this issue by using Amazon's Mechanical Turk (MTurk), which allowed us to recruit participants who were entirely naïve to the purposes of the research. We also changed the materials slightly such that the target character was given a gender-neutral name (Jessie) rather than a female name (Betty). Relatedly, Study 2 collected participant gender information, which was absent in the Study 1 protocol, to determine whether gender mattered in the analyses. Irrespective of these changes, we again hypothesized that participants with a growth mindset, relative to a fixed mindset, would express more negative attitudes towards a target person described as obese.

\section{Method}

\section{Participants and Design}

MTurk, which is a crowdsourcing site with thousands of individuals, was used to collect the data. Participants from MTurk have been shown to be more demographically 
diverse than typical university student samples and data obtained through MTurk have been shown to be as reliable as data obtained in the laboratory (Buhrmester, Kwang, \& Gosling, 2011; Meier \& Lappas, 2016). Potential participants were eligible if they were over 18 years old and lived in the United States. Compensation was \$.25.

The study collected data from 415 participants. However, rigorous exclusionary criteria reduced this number: 45 people were eliminated because they indicated that they had completed items used in the study before, 39 people were eliminated because they failed an attention check (see below), and 1 person was eliminated because gender information was missing. The remaining sample consisted of 330 participants, 142 of whom were female, who had a mean age of $33.74(S D=11.12)$. The majority of the sample $(81.2 \%)$ was Caucasian in race. As in Study 1, a computer algorithm randomly assigned participants to either obese or non-obese target conditions.

\section{Materials and Procedure}

Participants read a vignette about an obese or non-obese person and completed an assessment of negative attitudes (AFQ) followed by the STQ and demographic questions. The vignette was similar to the one used in Study 1, but was modified in two ways. Some of the language was changed from British English to American English given the change in sample demographics. Also, several changes were made to achieve gender neutrality. Gendered pronouns were not used and the target character's name was changed. In order to find a gender-neutral name, 41 participants not included in Studies 1 or 2 rated the extent to which nine names (Casey, Alex, Bailey, Riley, Jamie, Blake, Corey, Finley, and Jessie) were associated with a male or a female $(1=$ Very Male to $5=$ Neither Male nor Female to $9=$ Very Female). The average rating for "Jessie" was closest to the mid-point of the scale $(M=$ 4.98) and was chosen for use in the current study. A modified anti-fat questionnaire (the AFQ: Crandall, 1994) was again used to assess negative attitudes towards the fictional 
character $(M=3.62 ; S D=1.61 ; \alpha=.94)$ and Burnette's (2010) STQ assessed incremental theories about bodily weight $(M=4.77 ; S D=0.91 ; \alpha=.92)$.

After completing the measures, participants were asked to list the name of the person in the vignette and if they had completed a similar study in the past. Participants failing the first check were removed from the study and participants answering yes to the second question were removed as well. Procedures of this type constitute good practice (Buhrmester et al., 2011).

\section{Results}

As in Study 1, we calculated participant BMI scores $(M=27.20 ; S D=6.85 ; 7$ participants did not report height or weight) and examined them as a function of vignette condition. BMI scores did not differ by Weight Condition, $F(1,321)=.82, p=.37, \eta_{\mathrm{p}}^{2}<.01$.

To determine whether a growth mindset about weight, versus a fixed one, mattered in the person-perception paradigm, we performed a multiple regression. The dependent measure consisted of attitudes on the AFQ and the $Z$-scored predictors included weight condition, STQ scores, participant gender, and all 2- and 3-way interactions among these variables. In a replication of Study 1 , the obese target person $(M=3.96 ; S D=1.57)$ was evaluated more negatively than the non-obese target person $(M=3.20 ; S D=1.58), \beta=.23, t(329)=4.40, p<$ .01. Additionally, overall, males rated the target person more negatively $(M=3.85 ; S D=$ 1.60) than females $(M=3.31 ; S D=1.58), \beta=-.17, t(329)=-3.22, p<.01$. There was also an interaction between Gender and STQ scores, $\beta=-.12, t(329)=-2.17, p=.03$, with estimated means displayed in Figure 2. Male participants with higher STQ scores tended to make more negative evaluations, whereas female participants with higher STQ scores tended to make less negative evaluations. Given that the interaction did not include the weight condition variable, however, further interpretation would be speculative. 
Insert Figure 2

Of more importance, we were able to replicate the Weight Condition by STQ interaction of Study $1, \beta=.19, t(329)=3.49, p<.01$. Estimated means $(+/-1 S D)$ for this interaction are displayed in Figure 3 and the pattern was substantially the same as in the first study. When the target was obese, people with a growth mindset had more negative attitudes towards the target character, $\beta=.17, t(329)=2.23, p=.03$. By contrast, when the target was non-obese, people with a growth mindset had less negative attitudes towards the target character, $\beta=-.22, t(329)=-2.91, p<.01$. Thus, growth-minded people could be considered less tolerant of obesity than those with fixed theories of this attribute.

\section{Insert Figure 3}

As in Study 1, additional analyses revealed that people with a growth mindset $(+1 S D)$ expressed more negative attitudes towards the obese target relative to the non-obese target, $\beta$ $=.42, t(329)=5.68, p<.01$. By contrast, the weight manipulation did not affect people with low (-1 SD) STQ scores, who had a more fixed mindset concerning weight, $\beta=.04, t(329)=$ $.56, p=.58$. The remaining effects and interactions were not significant: STQ scores $-\beta=-$ $.03, t(329)=-.60, p=.55$, sex by obesity condition interaction $-\beta-.02, t(329)=-.40, p=.69$, and the sex by obesity condition by STQ scores interaction $-\beta=<.01, t(329)=.07, p=.94$. Overall, then, Study 2 replicated the key findings of Study 1 in the context of a larger sample size of individuals from a different country. A growth mindset concerning weight, relative to a fixed mindset, can result in more negative attitudes towards an obese person.

\section{General Discussion}


Obese people report themselves to be the frequent target of negative attitudes and discrimination (Puhl \& Brownell, 2001). The present studies sought to understand a class of variables that likely contribute to such forms of mistreatment. When people think that a given problem is controllable, not controlling the problem could be taken as a sign of personal weakness or deficiency, increasing the risk of harsh and negative judgments. Obesity is a realm in which such dynamics could operate because some believe that obese people could control the factors that give rise to their condition, perhaps by cutting down on food intake or eating healthier (Crandall, 1994). In a somewhat paradoxical fashion, then, people who have more optimistic ideas about weight control could hold more negative attitudes towards others whose weight seems out of control.

We used these ideas to probe for a potential downside to incremental theorizing. Generally, people with a growth mindset - who believe that attributes and conditions are malleable - are advantaged relative to others who believe that the same attributes are fixed (Dweck, 2000). In many cases, these advantages should directly follow from control beliefs, which tend to have both emotional and behavioural benefits, especially over time (Bandura, 1989). At the same time, however, these very same beliefs about controllability could result in less tolerance for other people who seem to have abdicated their responsibilities in the domain in question (Weiner, 1986). Through processes of this type, incremental theorizing could be linked to greater likelihood of negative attitudes. We found support for these ideas in that incremental theorizing about weight, relative to entity theorizing, led to negative attitudes towards a target that was described as obese. This antipathy encompassed both disliking and the sorts of discriminatory intentions captured by Crandall's (1994) anti-fat questionnaire.

Our project is not without limitations. One, we failed to assess participant demographic characteristics like gender in Study 1. Even so, we collected this information in 
Study 2 and gender did not modify the critical (weight condition by mindset) interaction in this second study, suggesting that the processes of interest seem to apply equally well to male and female theorizers. Two, our studies relied on a vignette method to investigate attitudes towards an obese person. The vignette method has some limitations and we therefore advocate extensions that attempt to measure negative attitudes or behaviour in more realistic settings. Such research can be difficult to conduct but would be helpful in further establishing the generalizability of the findings. Finally, one could assess attributions of blame, control, etc., to determine whether they mediate the impact of incremental theorizing on negative attitudes towards obese persons, in a manner consistent with attribution theory (Weiner, 1986) or system justification theory (Jost, et al., 2004). That is, it could be useful to find out more about the sorts of inferences that incremental and entity theorists make when they are exposed to targets that could be stigmatized.

Despite these limitations, the results of the current studies should not be understated. The concept of growth mindset in lay culture has received widespread positive attention given the impact that incremental theorizing can have on behaviour (Dweck, 2015). Indeed, even in this context, the few studies that have investigated the relationship between mindset and negative attitudes / prejudice / stigma seem to suggest that having a growth mindset makes individuals more empathetic to the troubles of others (Molden \& Dweck, 2006; Plaks, Levy \& Dweck, 2009). However, a very recent investigation reports results that reflect those of the current study. Ryazanov and Christenfeld (2018) found that individuals with a growth mindset exhibited higher levels of blame when imagining someone who continually displayed deficiencies in empathy, motivation and aggression. These researchers suggest adopting a contextual view when considering the impact of growth mindset on negative attitudes. Specifically, in some contexts, especially those where the trait is viewed to be in the control of the person in question, having a growth mindset will likely lead to greater 
prejudice. In our study, the person in question was someone presenting with obesity.

However, the principles should also be relevant to other domains in which a person could be accorded some control over an unfortunate outcome - such as poverty, criminality, or drug addiction. With respect to these sorts of outcomes, too, we might expect people with a growth mindset, relative to a fixed mindset, to hold others more accountable for the behaviours that presumably gave rise to the problem, exhibiting greater negative attitudes as a result. Even so, incremental theorists could be less prejudiced in the context of non-controllable, nonbehavioural features of the person like race or ethnicity (Levy et al., 1998). Future research should map these contingencies more fully, as doing so may lead to new insights into how implicit theorizing gives rise to perceptions of other people, in addition to the groups they belong to. For now, what we emphasize is that we have identified a domain - obesity - in which incremental theorizing seems to result in greater rather than lesser negative attitudes.

Overall, our findings qualify the idea that incremental theorizing has no downsides. For example, consider weight reduction programs in this context. A primary goal of many weight loss programs will be to create a growth mindset about weight. That is, people typically need to believe that weight is controllable before they will commit to substantial weight reduction goals (Schwarzer, 2001). Nonetheless, the same beliefs that could benefit the self are likely to result in negative attitudes towards others who are obese yet seemingly passive about the condition. Although the basis for such reactions is cognitive, considerable antipathy can result, as the present findings show. It might therefore be useful to supplement weight loss programs targeting ideas about malleability with interventions (such as compassion) that are helpful in reducing stigma (Luoma \& Platt, 2015). Of course, it is likely that such interventions would not only be of benefit to those on the receiving end of negative attitudes, but also to those holding them, given that our perspectives of others often impact our perspectives about ourselves (McHugh, Stewart, \& Hooper, 2012). 


\section{References}

Aiken, L. S., \& West, S. G. (1991). Multiple regression: Testing and interpreting interactions. Thousand Oaks, CA: Sage Publications.

Bandura, A. (1989). Human agency in social cognitive theory. American Psychologist, 44, $1175-1184$.

Biddle, S. J. H., Wang, C. K. J., Chatzisarantis, N. L. D., \& Spray, C. M. (2003). Motivation for physical activity in young people: Entity and incremental beliefs about athletic ability. Journal of Sports Sciences, 21, 973-989.

Buhrmester, M., Kwang, T., \& Gosling, S. D. (2011). Amazon's Mechanical Turk: A new source of inexpensive, yet high-quality, data? Perspectives on Psychological Science, 6, 3-5.

Burnette, J. L. (2010). Implicit theories of body weight: Entity beliefs can weight you down. Personality and Social Psychology Bulletin, 36, 410-422.

Crandall, C. S. (1994). Prejudice against fat people: Ideology and self-interest. Journal of Personality and Social Psychology, 66, 882-894.

Dweck, C. S. (2000). Self-theories: Their role in motivation, personality, and development. Hove, East Sussex, UK: Psychology Press.

Dweck, C. (2015). Carol Dweck revisits the 'growth mindset'. Education Week, 35, 20-24.

Dweck, C. S., \& Leggett, E. (1988). A social-cognitive approach to motivation and personality. Psychological Review, 95, 256-273.

Goodman, N., Richardson, S. A., Dornbusch, S. M., \& Hastorf, A. H. (1963). Variant reactions to physical disabilities. American Sociological Review, 28, 429-435.

Hegarty, P., \& Golden, A. M. (2008). Attributional beliefs about the controllability of stigmatized traits: Antecedents or justifications of prejudice? Journal of Applied Social Psychology, 38, 1023-1044. 
Jost, J. T., Banaji, M. R., \& Nosek, B. A. (2004). A decade of system justification theory: Accumulated evidence of conscious and unconscious bolstering of the status quo. Political psychology, 25(6), 881-919.

Kraut, R., Olson, J., Banaji, M., Bruckman, A., Cohen, J., \& Couper, M. (2004). Psychological research online: Report of board of Scientific Affairs' Advisory Group on the conduct of research on the Internet. American Psychologist, 59, 105-117.

Levy, S. R., Stroessner, S. J., \& Dweck, C. S. (1998). Stereotype formation and endorsement: The role of implicit theories. Journal of Personality and Social Psychology, 74, 14211436.

Luoma, J. B., \& Platt, M. G. (2015). Shame, self-criticism, self-stigma, and compassion in Acceptance and Commitment Therapy. Current opinion in psychology, 2, 97-101.

Meier, B. P., \& Lappas, C. M. (2016). The influence of safety, efficacy, and medical condition severity on natural versus synthetic drug preference. Medical Decision Making, 36, 1011-1019.

McHugh, L., Stewart, I. \& Hooper, N. (2012). A contemporary functional analytic account of perspective taking. In McHugh \& Stewart (Ed), The Self and Perspective Taking; Contributions from Contemporary Behavioral Science. ISSN: 157224995.

Molden, D. C., \& Dweck, C. S. (2006). Finding" meaning" in psychology: a lay theories approach to self-regulation, social perception, and social development. American Psychologist, 61(3), 192.

Plaks, J. E., Levy, S. R., \& Dweck, C. S. (2009). Lay theories of personality: Cornerstones of meaning in social cognition. Social and Personality Psychology Compass, 3(6), 10691081.

Puhl, R., \& Brownell, K. D. (2001). Bias, discrimination, and obesity. Obesity Research, 9, 788-805. 
Puhl, R., \& Brownell, K. D. (2003a). Psychosocial origins of obesity stigma: Toward changing a powerful and pervasive bias. Obesity Reviews, 4, 213-227.

Puhl, R., \& Brownell, K. D. (2003b). Ways of coping with obesity stigma: Review and conceptual analysis. Eating Behaviors, 4, 53-78.

Rubin, Z., \& Peplau, L. A. (1975). Who believes in a just world? Journal of Social Issues, 31, 65-89.

Ryazanov, A. A., \& Christenfeld, N. J. S. (2018). Incremental mindsets and the reduced forgiveness of chronic failures. Journal of Experimental Social Psychology, 76, 3341.

Schwarzer, R. (2001). Social-cognitive factors in changing health-related behaviors. Current Directions in Psychological Science, 10, 47-51.

Simons, D. J. (2014). The value of direct replication. Perspectives on Psychological Science, 9, 76-80.

Weiner, B. (1986). An attributional theory of achievement motivation and emotion. In An attributional theory of motivation and emotion (pp. 159-190). Springer US.

Weiner, B., Perry, R. P., \& Magnusson, J. (1988). An attributional analysis of reactions to stigmas. Journal of Personality and Social Psychology, 55, 738-748.

World Health Organisation, WHO. "Obesity and Overweight." Mar.-Apr. 2013. Retrieved 23.02.14 from http://www.who.int/gho/ncd/risk_factors/overweight/en/ 


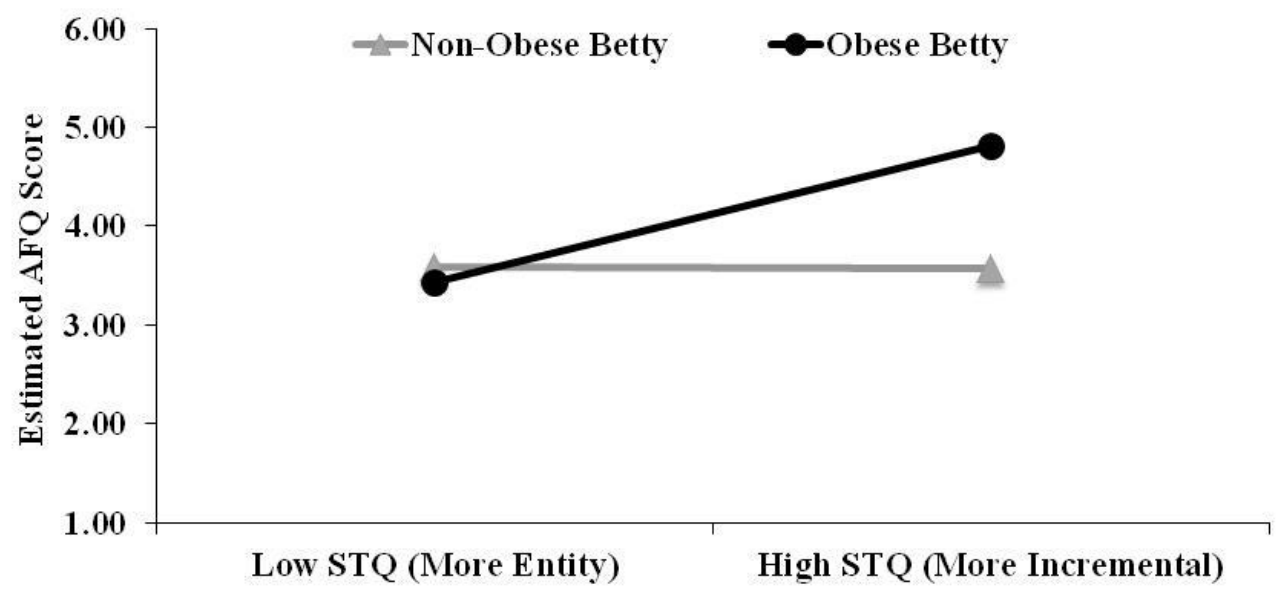

Figure 1. Estimated AFQ Scores for the Interaction Between Weight Condition and the STQ Continuum (+/- $1 S D$ from the $M)$, Study 1. Note: Higher AFQ scores reflect more negative attitudes and higher STQ scores represent more incremental beliefs about weight. 


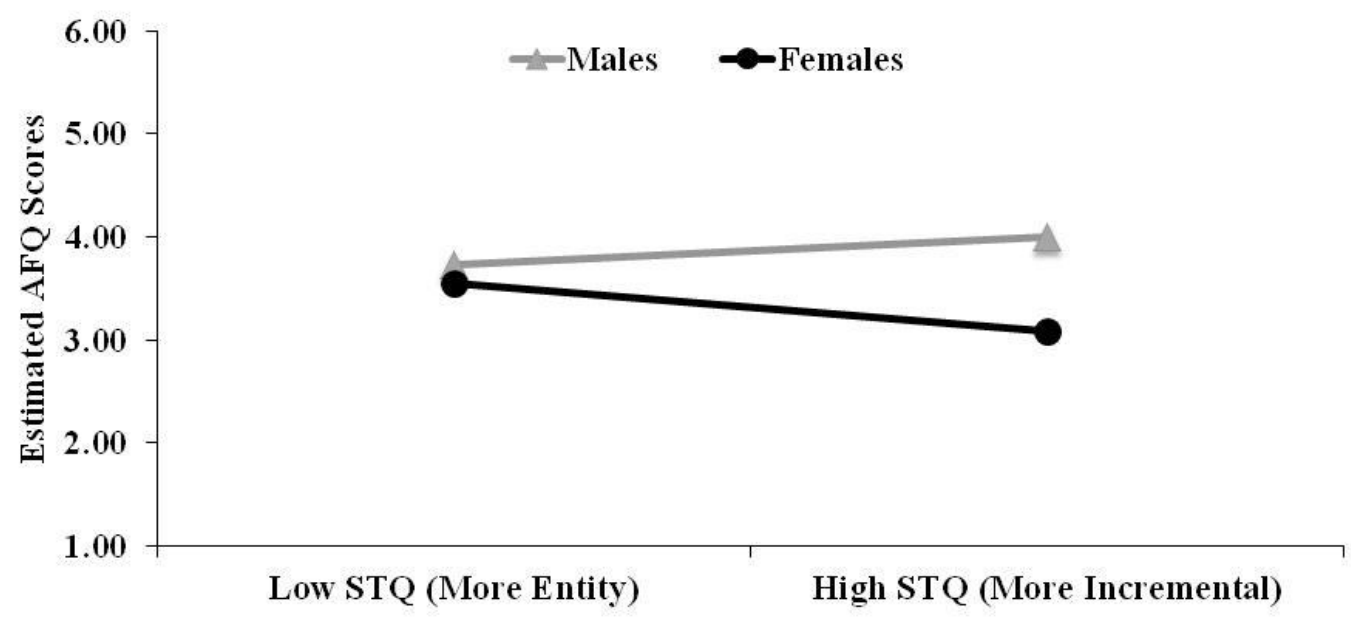

Figure 2. Estimated AFQ Scores for the Interaction Between Gender and the STQ Continuum (+/- $1 S D$ from the $M)$, Study 2. Note: Higher AFQ scores reflect more negative attitudes and higher STQ scores represent more incremental beliefs about weight. 


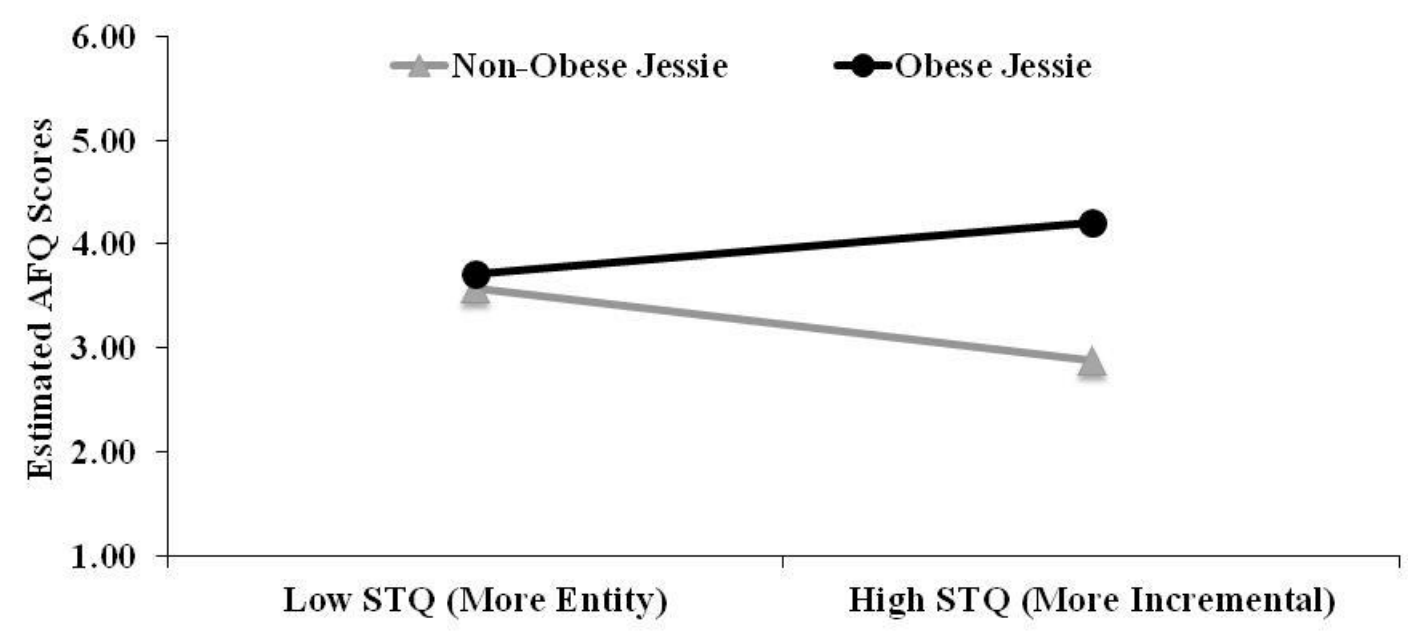

Figure 3. Estimated AFQ Scores for the Interaction Between Weight Condition and the STQ Continuum (+/- $1 S D$ from the $M)$, Study 2. Note: Higher AFQ scores reflect more negative attitudes and higher STQ scores represent more incremental beliefs about weight. 


\section{Appendix}

\section{Vignettes Used in Study 1}

Obese Condition: Betty is a 42-year-old woman who has been married to her husband for 15 years. Throughout most of her twenties she worked in temporary office jobs but now has a successful career in marketing. Betty has struggled with her weight her whole life but after a recent visit to her GP was found to have a BMI of 32 and diagnosed as clinically obese. Her general health is good and in her spare time she enjoys working on her allotment. Betty has three teenage children who all attend the local secondary school. She and her husband are hoping to book a holiday to Greece for the coming summer.

Non-Obese Condition: Betty is a 42-year-old woman who has been married to her husband for 15 years. Throughout most of her twenties she worked in temporary office jobs but now has a successful career in marketing. Betty has recently been to visit her GP who found that she had no significant medical problems and informed her that generally, her health is good. In her spare time she enjoys working on her allotment. Betty has three teenage children who all attend the local secondary school. She and her husband are hoping to book a holiday to Greece for the coming summer.

\section{Vignettes Used in Study 2}

Obese Condition: Jessie is a 42-year-old person who has been married for 15 years. Throughout most of Jessie's twenties, Jessie worked in temporary office jobs but now has a successful career in marketing. Jessie has always struggled with weight but after a recent visit to a physician, Jessie was found to have a BMI of 32 and was diagnosed as clinically obese. Jessie's general health is good. Jessie enjoys engaging in hobbies when free time is available. Jessie has three teenage children who all attend the local school. Jessie is hoping to book book a vacation to Greece for the coming summer. 
Non-Obese Condition: Jessie is a 42-year-old person who has been married for 15 years. Throughout most of Jessie's twenties, Jessie worked in temporary office jobs but now has a successful career in marketing. Jessie has recently been to visit a physician who found no significant medical problems and informed Jessie that generally, Jessie's health is good. Jessie enjoys engaging in hobbies when free time is available. Jessie has three teenage children who all attend the local school. Jessie is hoping to book book a vacation to Greece for the coming summer. 